[0212-7199 (2003) 20: 11; pp 585-593] Copyright @ 2003 ARAN EDICIONES, S.L

AN. MED. InTERNA (Madrid) Vol. 20, N. ${ }^{\circ} 11$, pp. 585-593, 2003

\title{
Trastornos metabólicos relacionados con el VIH y el tratamiento antirretroviral
}

\author{
B. ROCA
}

Unidad de Enfermedades Infecciosas. Hospital General. Castellón

METABOLIC DISORDERS ASSOCIATED WITH HIV AND ANTIRETROVIRAL THERAPY

\section{RESUMEN}

En los últimos años, gracias al tratamiento antirretroviral existente en la actualidad, el pronóstico de la infección por el VIH ha mejorado sustancialmente. Como consecuencia de ello, otros problemas clínicos están adquiriendo una importancia creciente en el control de los pacientes afectados por dicho virus. En este artículo se revisan las alteraciones metabólicas que ocasiona el propio VIH, y las que provoca el tratamiento antirretroviral.

PALABRAS CLAVE: VIH. Trastornos metabólicos. Tratamiento antirretroviral.

\section{ABSTRACT}

In the last few years, recently developed antirretroviral treatment has allowed a dramatic improvement in the prognosis of HIV disease. As a result, other medical problems are taking increasing relevance in the follow up of the patients infected by such virus. In this article, metabolic disorders associated with HIV, as well as those provoked by antiretroviral therapy, are comprehensively reviewed.

KEY WORDS: HIV. Metabolic disorders. Antirretroviral therapy.

Roca B. Trastornos metabólicos relacionados con el VIH y el tratamiento antirretroviral. An Med Interna (Madrid) 2003; 20: 585-593.

\section{INTRODUCCIÓN}

Con el tratamiento antirretroviral de gran actividad (TARGA) existente en la actualidad se ha logrado una considerable reducción de la morbilidad y la mortalidad relacionadas con el VIH (1). Como consecuencia de ello, otros problemas clínicos han ido adquiriendo una relevancia creciente en los pacientes con infección por dicho virus. Entre estos nuevos problemas ocupan un lugar prominente diversos trastornos metabólicos, generalmente provocados por los fármacos, como las dislipemias, la lipodistrofia, la intolerancia a la glucosa o el síndrome de la elevación del ácido láctico (2).

Estos trastornos con frecuencia se presentan asociados, y tienen un impacto negativo en el bienestar de los pacientes y en la adherencia al tratamiento antirretroviral. Existe además el temor de que esos cambios metabólicos puedan provocar una epidemia de enfermedades cardiovasculares, en un futuro más o menos lejano, en dichos pacientes (3). A la vista de ello, es necesario incluir en la atención de la enfermedad por el VIH el adecuado diagnóstico y tratamiento de tales procesos, así como el control de los otros factores de riesgo cardiovascular.

A continuación describimos los conocimientos existentes en la actualidad sobre cada uno de esos trastornos metabólicos (4).

\section{DISLIPEMIAS}

\section{SIGNIFICADO CLÍNICO Y EVALUACIÓN}

En las primeras fases de la infección por el VIH, cuando los pacientes se hallan completamente asintomáticos, puede producirse un descenso de los niveles de colesterol HDL. Años más tarde, cuando aparecen las manifestaciones clínicas relacionadas con el VIH, con frecuencia se observa un aumento de los niveles de triglicéridos y de colesterol LDL, y de

Trabajo aceptado: 26 de junio de 2003

Correspondencia: Bernardino Roca Villanueva. Cataluña, 33-A, 4. 12004 Castellón. e-mail: brocav@meditex.es. 
nuevo un descenso del colesterol HDL (5). Se trata en general de alteraciones proaterogénicas, aunque se desconocen sus auténticas repercusiones clínicas. Se han propuesto varias teorías para tratar de explicar estas dislipemias, pero hasta ahora se desconoce su verdadera etiopatogenia (6).

Los inhibidores de la proteasa (IP) son uno de los principales grupos de antirretrovirales (Tabla I), y con frecuencia forman parte del TARGA. Estos fármacos pueden aumentar considerablemente de los niveles de triglicéridos y de colesterol LDL. Especialmente comunes son estos efectos secundarios con ritonavir, uno de los constituyentes del grupo (7). El mecanismo por el que se producen estos trastornos lipídicos es desconocido; algunos autores creen que podrían estar relacionados con la reconstitución del sistema inmunológico que induce el TARGA, y otros consideran que podrían deberse a la inhibición por parte de los IP del metabolismo celular de los lípidos (8). Estos medicamentos no parecen modificar los niveles de colesterol HDL (9).

En la mayoría de pacientes el incremento de los niveles de lípidos es discreto, y probablemente sólo representa un ligero aumento del riesgo de arteriosclerosis. Este efecto adverso parece insignificante comparado con el beneficio que supone el adecuado control de la infección por el VIH. Sin embargo, en algunos enfermos se producen acusadas elevaciones de los triglicéridos y también, aunque en menor medida, del colesterol, lo cual puede tener una mayor trascendencia. En estos casos, especialmente cuando se suman otros factores de riesgo cardiovascular, la adecuada intervención es fundamental.

La hipertrigliceridemia también puede favorecer la aparición de pancreatitis. El riesgo de esta enfermedad es especialmente alto en pacientes que toman además otros fármacos capaces de causar por si mismos pancreatitis, como pentamidina. sulfametoxazol-trimetoprim o didanosina. Este último es un antirretroviral del grupo de los inhibidores de la transcriptasa análogos de los nucleósidos (ITAN) (Tabla I).

Los antirretrovirales de grupos diferentes a los IP no parecen producir trastornos relevantes en los lípidos (10).

\section{TRATAMIENTO}

Existen guías detalladas para el adecuado tratamiento de las distintas dislipemias en la población general (Tablas II y III) (11). En líneas generales, esas recomendaciones son adecuadas también para los pacientes con infección por el VIH. Sin embargo para estos enfermos son pertinentes algunas consideraciones específicas, que comentamos a continuación (12).

En todos los pacientes con infección por el VIH deben controlarse periódicamente los niveles de lípidos. Esta recomendación es especialmente importante en quienes toman IP $\mathrm{y}$ en quienes presentan dislipemias antes de iniciar el tratamiento (13).

En algunos pacientes con infección por el VIH no son aplicables las recomendaciones dietéticas contra las dislipemias que se emplean en la población general. Por ejemplo, hay enfermos con caquexia y dislipemia, a los que obviamente no se les puede recomendar que pierdan peso. Por otra parte, las interacciones de los alimentos con los fármacos también pueden dificultar el seguimiento de las dietas recomendables para las dislipemias. Así, el saquinvavir debe tomarse con alimentos ricos en grasas para mejorar su absorción, y el ritonavir es mejor tomarlo con alguna comida para

TABLA I

RESUMEN DE LAS CARACTERÍSTICAS DE LOS ANTIRRETROVIRALES

\begin{tabular}{lll}
\hline Fármaco & Dosis habitual & Principales efectos secundarios \\
\hline
\end{tabular}

Inhibidores de la transcriptasa análogos de los nucleósidos (ITAN) a

Zidovudina (AZT)

Didanosina (ddl)

Zalcitabina (ddC)

Estavudina (d4T)

Lamivudina (3TC)

Abacavir
250-300 mg / $12 \mathrm{~h}$

$400 \mathrm{mg}(250 \mathrm{mg}$ si peso < $60 \mathrm{Kg}) /$ día

$0,75 \mathrm{mg} / 8 \mathrm{~h}$

$40 \mathrm{mg}$ (30 mg si peso $<60 \mathrm{Kg}$ ) / $12 \mathrm{~h}$

$150 \mathrm{mg} / 12 \mathrm{~h}$

$300 \mathrm{mg} / 12 \mathrm{~h}$
Anemia, macrocitosis, neutropenia, miopatía Pancreatitis, neuropatía periférica Neuropatía periférica, úlceras orales Neuropatía periférica, lipoatrofia Escasos

Reacción de hipersensibilidad

\section{Astenia, náuseas}

Tenofovir

$300 \mathrm{mg} /$ día

Inhibidores de la transcriptasa no análogos de los nucleósidos (ITNN) ${ }^{\mathrm{b}}$

Nevirapina

$200 \mathrm{mg} / 12 \mathrm{~h}$

Efavirenz

$600 \mathrm{mg} /$ día

Inhibidores de la proteasa (IP) ${ }^{\mathrm{b}, \mathrm{c}}$

Saquinavir

Ritonavir

Indinavir

Nelfinavir

Amprenavir

Lopinavir - ritonavir
$1200 \mathrm{mg} / 8 \mathrm{~h}$

$600 \mathrm{mg} / 12 \mathrm{~h}$

$800 \mathrm{mg} / 8 \mathrm{~h}$

$750 \mathrm{mg} / 8 \mathrm{~h}$

$1200 \mathrm{mg} / 12$ horas

$300 \mathrm{mg}-100 \mathrm{mg} / 12$ horas
Erupciones cutáneas

Erupciones cutáneas, confusión, mareo

Diarrea, náuseas, cefalea Vómitos, diarrea, parestesias, etc. Hiperbilirrubinemia, nefrolitiasis Diarrea

Diarrea, vómitos

Diarrea, astenia

a Todos los medicamentos de estos grupos pueden causar el síndrome de la elevación del ácido láctico.

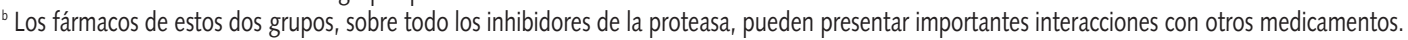

'Todos los medicamentos de este grupo pueden causar lipodistrofia, dislipemia e hiperglicemia. 
TABLA II

RECOMENDACIONES PARA EL CONTROL DEL COLESTEROL LDL Y DEL COLESTEROL NO-HDL EN ADULTOS DE LA POBLACIÓN GENERAL, ADAPTADAS DE LAS GUÍAS ATP III (11) (TODOS LOS LÍPIDOS MEDIDOS EN mg/dL)

Factores de riesgo Cifras de colesterol LDL deseables a Cifras de colesterol LDL a partir de las cuales Cifras de colesterol LDL a partir de las cardiovascular se recomienda dieta y ejercicio ${ }^{\text {a }} \quad$ cuales puede estar indicado el tratamiento farmacológico ${ }^{\text {a }}$

\begin{tabular}{llll}
\hline Cardiopatía isquémica o equivalentes $^{b}<100$ & $\geq 100$ & $\geq 130$ \\
2 o más factores de riesgo ${ }^{c}$ & $<130$ & $\geq 130$ & $\geq 160$ \\
$0-1$ factores de riesgo $^{c}$ & $<160$ & $\geq 160$ & $\geq 190$ \\
\hline
\end{tabular}

a Para el colesterol no HDL (colesterol total - colesterol HDL) las cifras deseables son $30 \mathrm{mg} / \mathrm{dL}$ mayores que para el colesterol LDL.

${ }^{\circ}$ Equivalentes de la cardiopatía isquémica: estenosis corotídea sintomática, arteriopatía periférica y aneurisma de la aorta abdominal.

' Factores de riesgo cardiovascular: tabaquismo, hipertensión, cifras bajas de colesterol HDL, historia familiar de cardiopatía isquémica prematura, y edad > 45 años en varones 0 > 55 en mujeres.

TABLA III

RECOMENDACIONES PARA EL CONTROL DE LOS TRIGLICÉRIDOS EN ADULTOS DE LA POBLACIÓN GENERAL, ADAPTADAS DE LAS GUÍAS ATP III (11) (TODOS LOS LÍPIDOS MEDIDOS EN mg/dL)

\begin{tabular}{ll}
\hline Cifras de triglicéridos & Recomendaciones \\
\hline $150-199$ & El objetivo principal es el control del \\
& colesterol LDL \\
& Dieta si existe sobrepeso \\
& Ejercicio físico \\
& Añadir a las recomendaciones \\
& anteriores un fibrato, ácido nicotínico \\
& u otro hipolipemiante \\
$200-499$ & Añadir a las recomendaciones \\
& anteriores una dieta muy baja en \\
& grasas ( $\leq 15 \%$ de las calorías en \\
& grasas)
\end{tabular}

minimizar los trastornos gastrointestinales que produce. Todas estas circunstancias claramente dificultan el seguimiento de cualquier dieta. En estos casos los consejos de un dietista pueden ser útiles para conseguir un equilibrio entre la ingesta calórica adecuada, el tratamiento de la dislipemia y el tratamiento de la infección por el VIH.

La disfunción hepática es común en los pacientes con infección por el VIH. Ello puede deberse a numerosos factores, como la gran prevalencia de coinfección por los virus de las hepatitis $\mathrm{C}$ o B, el etilismo crónico o la toxicidad de los fármacos. Como varios de los medicamentos que se emplean en el tratamiento de las dislipemias son también hepatotóxicos, se recomienda especial precaución si se emplean dichos fármacos en enfermos con infección por el VIH $(14,15)$.

Cuando aparece un efecto secundario de un medicamento, la mayoría de las veces la solución óptima es su retirada. Sin embardo, cuando se trata de un tratamiento esencial, antes de adoptar una decisión deben ponderarse bien los beneficios y los riesgos de mantenerlo. Esta situación es muy común con el TARGA. En los casos de dislipemia relacionados con los IP, caben varias posibilidades. Si la infección por el VIH está bien controlada y la dislipemia no es muy acusada, probablemente lo mejor es mantener el tratamiento y aconsejar dieta y ejercicio físico, si son adecuados para el paciente. Sin embar- go, si el control de la infección por el VIH es subóptimo, o la dislipemia es intensa o difícil de corregir, probablemente lo más adecuado es sustituir el IP por un medicamento de otro grupo $(16,17)$. Entre las alternativas a los IP figuran efavirenz y nevirapina, dos inhibidores de la transcriptasa no nucleósidos (ITNN), tenofovir, un inhibidor de la transcriptasa análogo de los nucleótidos (ITANt), y abacavir, un ITAN (Tabla I).

La hipertrigliceridemia no supone un riesgo cardiovascular de tanta magnitud como la hipercolesterolemia. De cualquier modo, debido al riesgo de pancreatitis que también conlleva, su control es aconsejable en todos los pacientes que la presenten. En enfermos con hipertrigliceridemia y diabetes, el control de la glucemia suele ayudar a reducir los niveles de triglicéridos (18). También es conveniente que los pacientes con hipertrigliceridemia eviten el alcohol, ya que su ingesta tiende a incrementar los niveles de dichas grasas.

La hipercolesterolemia, incluso cuando es ligera, debe tratarse correctamente, ya que su poder aterogénico es muy alto. Entre las recomendaciones dietéticas figura la reducción de la ingesta de colesterol a menos de $200 \mathrm{mg}$ al día, la reducción de la ingesta de grasas saturadas a menos del $7 \%$ del total de calorías diarias y la reducción de las grasas totales a menos del $30 \%$ de las calorías totales diarias. Los alimentos con grasas insaturadas, como el aceite de oliva o las nueces, y el ejercicio físico son asimismo útiles para reducir los niveles de colesterol (19-21). En pacientes con hipercolesterolemia severa o con otros factores de riesgo cardiovascular asociados, es necesario seguir estrictamente ese tipo de recomendaciones, y además con frecuencia son necesarios también medicamentos hipolipemiantes.

Las estatinas son los fármacos más empleados en el tratamiento de las dislipemias. Son capaces de reducir los niveles de colesterol total, colesterol LDL y triglicéridos, y de aumentar los niveles de colesterol HDL. En la población general se ha demostrado que disminuyen la morbilidad y la mortalidad cardiovascular (22). Atorvastatina parece ser el compuesto más potente del grupo. Los IP inhiben el sistema enzimático del citocromo P-450, que se encuentra principalmente en el hígado. Por esa vía se metabolizan todas las estatinas, excepto pravastatina, que se elimina esencialmente por vía renal. Por ello, se desaconseja la utilización conjunta de los IP con simvastatina y lovastatina, y se recomienda precaución si dichos antivirales se emplean conjuntamente con las otras estatinas (23). Otros medicamentos de empleo frecuente en la infección por el VIH, como los antibióticos macrólidos y diversos anti- 
fúngicos, también pueden presentar interacciones relevantes con las estatinas.

El principal efecto secundario de las estatinas es la miopatía. Uno de los compuestos del grupo, cerivastatina, fue retirado del mercado por ocasionar cuadros de rabdomiólisis agudas, en algunos casos fatales, especialmente en enfermos que estaban tomando también fibratos (24). Cuadros de rabdomiólisis similares se han descrito con prácticamente todas las estatinas, por ese motivo se recomienda precaución al utilizarlas, y se desaconseja su empleo concomitante con fibratos (25). Las estatinas también pueden ocasionar elevación de las enzimas hepáticas, y deben evitarse en pacientes con hepatopatías.

Los fibratos pueden ser útiles en la hipertrigliceridemia. Entre ellos, el más recomendable en pacientes con infección por el VIH es probablemente el fenofibrato, ya que no se metaboliza a través del citocromo P-450, y por tanto las interacciones medicamentosas son menos probables que con otros preparados (26). No obstante, estos medicamentos con frecuencia ocasionan dispepsia, elevación de enzimas hepáticas $\mathrm{y}$ otros efectos secundarios, que restringen considerablemente su utilidad (27).

Otros grupos de hipolipemiantes son las resinas secuestradoras de ácidos biliares y el ácido nicotínico y sus derivados. Sin embargo la limitada eficacia y los frecuentes efectos secundarios de estos preparados hacen que su utilidad sea limitada en pacientes con otros problemas médicos, como la infección por el VIH (28).

\section{LIPODISTROFIA Y LIPOATROFIA}

\section{SIGNIFICADO CLÍNICO Y EVALUACIÓN}

Los pacientes que toman TARGA con frecuencia presentan alteraciones en la distribución de la grasa corporal. Entre ellas figura la lipohipertrofia o acúmulo de grasa en las vísceras, la pared abdominal, las mamas tanto del varón como de la mujer, y la parte posterior del cuello (cuello de búfalo); puede tratarse de un proceso generalizado o localizado. Otra modalidad de este proceso es la lipoatrofia o pérdida de la grasa subcutánea de la cara, los glúteos y las extremidades, que suele manifestarse con la aparición de unas venas mucho más prominentes de lo normal. Al conjunto de estos trastornos se le conoce con el nombre de lipodistrofia. Las distintas modalidades de redistribución de la grasa corporal pueden presentarse simultáneamente en un mismo paciente. Se desconoce la verdadera prevalencia de estos procesos, pero se cree que pueden afectar hasta a la mitad de los pacientes que toman TARGA de modo prolongado (29).

La lipodistrofia está relacionada principalmente con los IP (30), pero la lipoatrofia también se debe, al menos en parte, a los ITAN, grupo de fármacos utilizado en prácticamente todas las combinaciones de TARGA en la actualidad (31). Los ITNN no parecen influir sobre estos trastornos (32).

Las distintas modalidades de lipodistrofia son más frecuentes en pacientes de mayor edad, con infección por el VIH más avanzada, o con una mejor respuesta al tratamiento con TARGA. También son más comunes en mujeres que en hombres, y en personas de raza blanca que en las de otras razas (29). Factores hereditarios también pueden influir en su desa- rrollo (33). El tipo de lipodistrofia depende en parte de la constitución de cada paciente antes de iniciar el tratamiento. Así, las personas delgadas tienden a desarrollar lipoatrofia y las obesas lipohipertrofia.

Por ahora se desconocen los mecanismos fisiopatológicos que llevan al desarrollo de la lipodistrofia. Algunos estudios sugieren la participación de diversas citocinas (34), otros indican una posible alteración del metabolismo hepático de las grasas (35) y otros concluyen que los IP pueden actuar modificando la diferenciación de los preadipocitos (36). La lipoatrofia puede deberse a la inhibición de la ADN polimerasa gamma mitocondrial por parte de los ITAN (37). Se ha comprobado la existencia de apoptosis en los adipositos subcutáneos de los pacientes con lipoatrofia.

No existe ningún procedimiento sencillo y preciso que pueda recomendarse en la práctica clínica habitual para diagnosticar la lipodistrofia. La tomografía computarizada (TC) y la resonancia magnética (RM) son los métodos más fiables para medir la grasa corporal, tanto subcutánea como visceral. Con la realización de un único corte puede ser suficiente para obtener una evaluación adecuada (38). Pero por ahora no están bien definidos los valores normales y patológicos, en parte debido a que la distribución normal de la grasa corporal varía con la edad, el sexo y otros factores. En ocasiones resulta especialmente complicado diferenciar la lipoatrofia de la malnutrición (39).

Las medidas antropométricas, como la circunferencia de la cintura, el diámetro sagital o la relación cintura-cadera, son sencillas de determinar, pero tampoco se hallan bien definidos los valoren que permiten diferenciar lo normal de lo patológico (40).

La densitometría, basada en la medición de la absorción de rayos $\mathrm{X}$ por los tejidos, es útil para evaluar la grasa subcutánea de la parte distal de las extremidades, pero no proporciona resultados fiables en el abdomen (41). Otros métodos como la ecografía o la medición de la impedancia bioeléctrica son también imprecisos y de limitada utilidad.

La lipodistrofia habitualmente no ocasiona ningún problema clínico relevante, pero en algunos casos se ha asociado con sensación de disnea, cefalea, dolores localizados, reflujo gastroesofágico y otros síntomas inespecíficos (42).

\section{TRATAMIENTO}

En ausencia de otras complicaciones metabólicas asociadas, el tratamiento de la lipodistrofia parece innecesario desde el punto de vista clínico. Sin embargo, su efecto cosmético puede ser tan negativo en algunos pacientes, que su presencia nunca puede ser menospreciada (43).

Lógicamente, si se decide tratar el proceso, la primera medida a tomar es la supresión del medicamento responsable. En pacientes con acúmulo central de grasa que estén tomando IP es aconsejable suprimir estos fármacos, y sustituirlos por efavirenz, nevirapina, tenofovir o abacavir. Con ello parece que no se resuelve el proceso, pero al menos probablemente no avanza más (44). En pacientes con lipoatrofia que estén tomando estavudina, un ITAN, es aconsejable suprimir este medicamento, ya que ha sido el más relacionado con ese trastorno. Generalmente puede sustituirse por otro fármaco del mismo grupo, con lo que suele producirse una resolución parcial del proceso (45). No obstante existen muchas dudas en 
este terreno, y es posible que otros ITAN, e incluso el propio VIH, produzcan también lipoatrofia (46).

La dieta no parece influir en el desarrollo o la evolución de la lipodistrofia, por tanto, si no existen otros trastornos metabólicos asociados, no está indicado seguir ningún tipo de dieta en especial. No obstante, en pacientes lipohipertrofia, central o generalizada, sí que está indicado el ejercicio físico, ya que su realización puede reducir el tejido adiposo (47).

Diversos medicamentos se han ensayado en el tratamiento de la lipodistrofia. El déficit de andrógenos tiende a producir acúmulo de grasa visceral y resistencia a la insulina (48). En base a ello se han utilizado testosterona y otros andrógenos en la lipodistrofia, aunque por ahora no han demostrado eficacia alguna. La hormona del crecimiento en dosis suprafisiológicas es capaz de reducir los acúmulos de grasa, tanto abdominales como cervicales. Sin embargo su acción es transitoria, y además posee efectos secundarios, como la intolerancia a la glucosa. Por ello su utilización de modo generalizado no está recomendada (42).

Algunos estudios realizados en pacientes con infección por el VIH han demostrado cierta eficacia de la metformina en el tratamiento de la hiperglucemia y las dislipemias, y en la reducción de los depósitos de grasa visceral. Por ello, especialmente en pacientes con dichos trastornos metabólicos asociados, puede utilizarse este medicamento como tratamiento de la lipohipertrofia.

Las tiazolidindionas, como rosiglitazona o pioglitazona, parecen capaces de reducir la grasa visceral en pacientes con diabetes tipo 2 y con lipodistrofias de tipo hereditario. Sin embargo, por el momento no existen estudios en pacientes con infección por el VIH que demuestren su eficacia, y por tanto su empleo de modo generalizado no está recomendado.

Otra alternativa terapéutica para la lipodistrofia es la cirugía plástica. La liposucción es útil en muchos casos de acúmulos lipídicos, por ejemplo de la pared abdominal. Por otra parte, los implantes de grasa autóloga o de diversos materiales sintéticos, como la silicona o el ácido poliláctico, son útiles en la lipoatrofia. Los resultados iniciales de estos procedimientos son buenos en muchos casos, pero se desconoce la consistencia de los mismos a largo plazo (49).

\section{HIPERGLUCEMIA}

\section{SIGNIFICADO CLÍNICO Y EVALUACIÓN}

Antes de existir el TARGA, la hiperglucemia era poco frecuente en los pacientes con infección por el VIH, ya que se trataba de una población predominantemente joven en la que la prevalencia de diabetes es baja.

Sin embargo, en la actualidad, se observa intolerancia a la glucosa debida a resistencia a la insulina en hasta el $40 \%$ de quienes toman IP (18). Estos medicamentos también dificultan el control de la glucemia y favorecen la descompensación cetoacidósica en los enfermos que previamente tenían diabetes.

Otros medicamentos que pueden ocasionar hiperglucemia son los corticosteroides y la pentamidina, que se utilizan en el tratamiento de algunas infecciones oportunistas, el megestrol, que se emplea como estimulante del apetito en pacientes con enfermedad por el VIH, y la didanosina (50).

Se define como intolerancia a la glucosa la presencia de unos niveles de glucosa iguales o superiores a $140 \mathrm{mg} / \mathrm{dl}$ dos horas después de la administración de $75 \mathrm{~g}$ de glucosa por vía oral. Y se define como diabetes mellitus la presencia de unos niveles de glucemia basal iguales o superiores a $126 \mathrm{mg} / \mathrm{dl}$ o bien unos niveles iguales o superiores a $200 \mathrm{mg} / \mathrm{dl}$ dos horas después de la administración de $75 \mathrm{~g}$ de glucosa por vía oral.

Se desconoce la proporción de pacientes con intolerancia a la glucosa que desarrollarán diabetes al tomar IP, pero quienes presentan otros factores de riesgo para tener diabetes tipo 2, como obesidad, probablemente se hallan expuestos a un riesgo especialmente alto (51).

La resistencia a la insulina se caracteriza por una reducción de la captación de glucosa por parte del músculo y un aumento de la gluconeogénesis por parte del hígado. Se desconoce el mecanismo por el que los IP ocasionan resistencia a la insulina, pero se cree que pueden actuar inhibiendo la captación celular de glucosa (52). En personas con infección por el VIH la resistencia a la insulina con frecuencia se asocia con la lipohipertrofia central o visceral y las dislipemias producidas también por los IP. Más discutible es la asociación de la resistencia a la insulina con la lipoatrofia provocada por los ITAN.

La resistencia a la insulina, incluso sin diabetes, conlleva riesgo cardiovascular, tanto por sí misma, como por su asociación con factores como las dislipemias, la hipertensión y la trombosis (53).

\section{TRATAMIENTO}

En pacientes con infección por el VIH con antecedentes de cualquier trastorno del metabolismo de la glucosa, o con familiares de primer grado de parentesco con diabetes, se recomienda evitar el empleo de IP, en cuyo lugar puede utilizarse efavirenz, nevirapina, tenofovir o abacavir (54).

El tratamiento de la intolerancia a la glucosa y la diabetes es similar al empleado en la población general (55). La dieta equilibrada y el ejercicio físico son importantes, e incluso previenen la diabetes en pacientes con intolerancia a la glucosa. En personas con sobrepeso está indicado adelgazar hasta alcanzar el peso ideal.

Si con las medidas anteriores no es suficiente pueden utilizarse medicamentos, especialmente de aquellos que aumentan la sensibilidad a la insulina, como metformina o una tiazolidindiona (rosiglitazona o pioglitazona) (56). Si con ellos el control de la glucemia no es adecuado, puede emplearse insulina, bien sola o bien asociada a los mismos fármacos. Sin embargo, en pacientes con infección por el VIH, no son muy recomendables los medicamentos hipoglucemiantes de otros grupos, como las sulfonilureas o las glitinidas, que actúan estimulando la secreción de insulina.

Si se emplean medicamentos deben tenerse en cuenta sus efectos secundarios y sus posibles interacciones con otros fármacos que puedan estar tomando los pacientes. La metformina puede causar el síndrome de la elevación del ácido láctico, proceso de extremada gravedad que también pueden ocasionar los antirretrovirales, en especial los ITAN (57). En pacientes que tomen cualquiera de esos medicamentos, debe vigilarse la posible aparición de síntomas sugestivos de ese síndrome (véase más adelante). En general, en pacientes con niveles elevados de ácido láctico o de creatinina se desaconseja la utilización de metformina. Las precauciones deben ser máximas en quienes toman simultáneamente metformina e ITAN (58). 
Las tiazolidindionas pueden ocasionar disfunción hepática, por ello se recomienda realizar periódicamente análisis de las enzimas hepáticas a quienes tomen esos fármacos. En los pacientes con infección por el VIH existen con frecuencia otras posibles causas de disfunción hepática, como la coinfección por el virus de la hepatitis $\mathrm{C}$, o la toxicidad de otros medicamentos, por ello dicho control es especialmente pertinente. En general, en pacientes con elevaciones moderadas o importantes de las enzimas hepáticas se desaconseja la utilización de tiazolidindionas (59).

En lo que a interacciones se refiere, los IP, y en menor medida los ITNN, pueden influir sobre el metabolismo de los hipoglucemiantes, al actuar sobre el citocromo P450. Así, ritonavir, puede reducir los niveles de las sulfonilureas, y puede aumentar los niveles de diversas glitinidas (4).

\section{SÍNDROME DE LA ELEVACIÓN DEL ÁCIDO LÁCTICO}

\section{SIGNIFICADO CLÍNICO Y EVALUACIÓN}

Un porcentaje considerable de pacientes que toman HAART presentan elevación de los niveles séricos de ácido láctico, es decir, cifras superiores a $18 \mathrm{mg} / \mathrm{dl}$ ( $2 \mathrm{mmol} / \mathrm{l})$. En la mayoría de casos este trastorno no tiene repercusión clínica alguna. Sin embargo en un pequeño porcentaje de enfermos dicha alteración se acompaña de malestar general, astenia, anorexia, pérdida de peso, dolor abdominal, disnea, insuficiencia respiratoria y arritmias cardiacas. Con frecuencia este proceso se complica con insuficiencia hepática, que puede manifestarse con hepatomegalia dolorosa, ascitis, edemas o encefalopatía, si bien el aumento de la bilirrubina no es común y las enzimas hepáticas no suelen hallarse muy elevadas. Los estudios de imagen y la biopsia hepática muestran generalmente esteatosis, y en los casos más graves necrosis del parénquima hepático (60). En general, con cuadros clínicos más graves se observan niveles más altos de ácido láctico.

Este síndrome puede iniciarse de un modo agudo o subagudo, y con frecuencia está desencadenado por algún proceso intercurrente, especialmente por una infección. Ocurre en pacientes de todas las edades y de ambos sexos por igual, aunque puede ser más frecuente en mujeres gestantes. Está producido por los ITAN. Se ha relacionado especialmente con estavudina y zidovudina, pero todos los compuestos del grupo pueden producirlo, especialmente cuando se han tomado durante más de 6 meses (61). La ribavirina, comúnmente empleada en el tratamiento de la coinfección por el virus de la hepatitis C, parece favorecer su desarrollo (62).

En la etiopatogenia de este síndrome interviene la inhibición de la ADN polimerasa gamma de las mitocondrias por parte de los ITAN (63). Este proceso ocasionalmente se asocia con otros efectos secundarios de los antirretrovirales, como la lipoatrofia, la osteopenia o la neuropatía periférica. (64).

El diagnóstico del síndrome de la elevación del ácido láctico con frecuencia plantea dificultades, debido a la inespecificidad de sus síntomas y a la imposibilidad de prever su aparición. El diagnóstico diferencial debe realizarse con otras causas de insuficiencia hepática, como las hepatitis agudas o la toxicidad por medicamentos, con procesos infecciosos que cursan con sepsis, y con la pancreatitis aguda (65).

Para interpretar correctamente el resultado de los niveles de ácido láctico deben tenerse algunas precauciones. Antes de realizarse la extracción de sangre debe evitarse el ejercicio físico intenso durante al menos 24 horas. Al realizarse la extracción el estado de hidratación debe ser normal y debe evitarse la habitual maniobra de abrir y cerrar el puño con fuerza repetidamente. La sangre extraída debe depositarse rápidamente en un tubo previamente enfriado y debe trasportarse con hielo al laboratorio, donde se efectuará la determinación de ácido láctico antes de transcurridas cuatro horas. Un único resultado elevado debe interpretarse con precaución, y siempre conviene repetirlo antes de llegar a conclusiones definitivas (4). Un resultado confirmado superior a $45 \mathrm{mg} / \mathrm{dl}(5 \mathrm{mmol} / \mathrm{l})$ en un contexto clínico adecuado, o un resultado confirmado superior a $90 \mathrm{mg} / \mathrm{dl}(10 \mathrm{mmol} / \mathrm{l})$ aunque sea con una clínica inespecífica, pueden considerarse diagnósticos.

La determinación rutinaria de los niveles de ácido láctico no está justificada en el seguimiento habitual de los pacientes con enfermedad por el VIH. Sin embargo, si que conviene realizar este tipo de análisis en enfermos que estén tomando ITAN y que presenten clínica sugestiva, disminución de los niveles de bicarbonato, aumento del gap aniónico $(\mathrm{Na}-(\mathrm{Cl}+\mathrm{HCO} 3)$ >16), o disfunción hepática no atribuible a otras causas.

Cuando el cuadro clínico es intenso y los niveles de ácido láctico superan los $90 \mathrm{mg} / \mathrm{dl}$ el pronóstico es malo, incluso muchas veces fatal. Los cuadros menos intensos se resuelven bien, aunque los síntomas y las alteraciones analíticas pueden persistir bastantes semanas antes de normalizarse (30).

En este proceso no siempre existe acidosis, especialmente en los casos menos graves. Por tanto la denominación "acidosis láctica", comúnmente empleada para referirse a él, no parece muy adecuada.

\section{TRATAMIENTO}

Se han ensayado diversas vitaminas y antioxidantes en el tratamiento del síndrome de la elevación del ácido láctico, sin embargo ninguna de estas substancias ha demostrado ser eficaz. Por tanto, las únicas medidas a tomar son la supresión del tratamiento antirretroviral y las habituales medidas de sostén aplicables a cualquier proceso agudo. Una vez resuelto el cuadro clínico y normalizado el ácido láctico, puede reiniciarse el tratamiento, pero sin ITAN ni ITANt, con combinaciones que incluyan ITNN e IP. En pacientes que no han presentando cuadros clínicos muy intensos y cuyos niveles de ácido láctico no han superado los $45 \mathrm{mg} / \mathrm{dl}$, puede contemplarse la posibilidad de reintroducir modalidades de HAART con ITAN o ITANt. En estos casos se procurará utilizar compuestos de esos grupos diferentes a los que estaban usándose al comenzar el proceso, y se vigilará estrechamente una posible recidiva clínica y una posible nueva elevación de los niveles de ácido láctico. Para ello se recomienda realizar análisis mensuales durante varios meses.

\section{PATOLOGÍA ÓSEA}

\section{OSTEONECROSIS}

Tradicionalmente la osteonecrosis o necrosis avascular ósea se ha relacionado con distintos factores de riesgo, entre los que figuran el alcoholismo, la dislipemia, el lupus eritema- 
toso sistémico, la anemia de células falciformes, los síndromes de hipercoagulabilidad y el empleo de corticosteroides. La infección por el VIH se reveló como un factor de riesgo más de este proceso (66), y en los últimos años, algunos estudios han señalado que el tratamiento antirretroviral se asocia también con esa afección (67).

La osteonecrosis se debe a un déficit circulatorio de patogenia incierta, que afecta principalmente a la cabeza femoral, aunque también puede presentarse en la cabeza humeral, los cóndilos femorales, la parte proximal de la tibia, los huesos pequeños de las manos, etc. Puede ocurrir en un solo hueso o en varios, y con cierta frecuencia aparece en dos huesos simétricos. Se manifiesta principalmente con dolor localizado y con incapacidad funcional del área afectada, aunque muchas veces la sintomatología es poco llamativa. El diagnóstico se realiza mediante radiografías, y con más fiabilidad con RM o TC. Si se detecta osteonecrosis en un hueso, está indicado estudiar también el hueso contralateral. La sustitución del hueso afectado por una prótesis es el único tratamiento existente para esta enfermedad.

\section{OSTEOPOROSIS}

La osteoporosis es otro trastorno óseo que se ha relacionado con la infección por el VIH, y sobre todo con el tratamiento TARGA que incluye fármacos del grupo de los IP $(68,69)$. Se desconocen los mecanismos por los que se produce. Hasta ahora no se han comunicado muchos casos de fracturas en pacientes con infección por el VIH y osteoporosis, pero se desconoce qué puede ocurrir en el futuro con este trastorno (70).

El diagnóstico de la osteoporosis suele efectuarse mediante radiografías, TC o estudios de densitometría ósea. Aunque por ahora no está recomendado realizar estudios rutinarios de este tipo en el seguimiento habitual de los pacientes con infección por el VIH.

En los enfermos con osteoporosis deben buscarse otros posibles factores causantes de dicho proceso, como tirotoxicosis, hiperparatiroidismo, hipogonadismo, etilismo, malabsorción, inactividad física, pérdida de peso reciente, o utilización de medicamentos como corticosteroides, fenobarbital, pentamidina o ketoconazol. Siempre que resulte posible se corregirán estos factores. Además, todos los pacientes afectados deben ingerir una cantidad suficiente de calcio y vitamina $\mathrm{D}$, en forma de alimentos, o bien de suplementos de dichos elementos. En determinados casos pueden prescribirse también difosfonatos.

\section{Bibliografía}

1. Murphy EL, Collier AC, Kalish LA, Assmann SF, Para MF, Flanigan TP et al. Highly active antiretroviral therapy decreases mortality and morbidity in patients with advanced HIV disease. Ann Intern Med 2001; 135: 17-26.

2. Hirsch HH, Battegay M. Lipodystrophy Syndrome by HAART in HIVInfected Patients: Manifestation, Mechanisms and Management. Infection 2002; 30: 293-8.

3. Carr A, Cooper DA. Adverse effects of antiretroviral therapy. Lancet 2000; 356: 1423-30.

4. Schambelan M, Benson CA, Carr A, Currier JS, Dubé MP, Gerber JG et al. Management of Metabolic Complications Associated With Antire-

\section{OTROS PROCESOS}

\section{CAQUEXIA RELACIONADA CON EL VIH}

Antes de que existiera el TARGA, los pacientes con infección avanzada por el VIH con frecuencia presentaban un cuadro de caquexia de progresiva instauración, que solía conducir a la muerte. En estos pacientes muchas veces se hallaban elevados los triglicéridos y disminuido el colesterol HDL. Este cuadro probablemente está provocado por el propio VIH, y en su patogenia probablemente intervienen citocinas como la interleucina 2 y el factor de necrosis tumoral. En la actualidad esta entidad es mucho menos frecuente $(71,72)$, pero continúa observándose en pacientes que no reciben TARGA. Como tratamiento pueden utilizarse estimulantes del apetito, como megestrol, pero lo más eficaz de todo es el adecuado control de la infección por el VIH con el tratamiento antirretroviral (73).

\section{HIPOGONADISMO}

En la época anterior al TARGA, en pacientes con enfermedad por el VIH avanzada, también era común el hipogonadismo, tanto en varones como en mujeres. Se trataba de enfermos que con frecuencia presentaban además otros procesos como la caquexia relacionada con el VIH o el complejo demencia-sida. En la actualidad sigue observándose este trastorno en pacientes no tratados. En los varones se caracteriza por la disminución de los niveles de testosterona y manifestaciones clínicas como la disminución de la libido o la impotencia. En las mujeres se caracteriza por la disminución de los niveles de estradiol y manifestaciones clínicas como la infertilidad, la amenorrea y la disminución de la libido.

En la mayoría de casos se trata de un hipogonadismo hipogonadotropo, es decir con niveles FSH y LH disminuidos, y además con respuesta hipofisaria normal a la hormona liberadora de gonadotropinas. La prolactina suele estar aumentada. Todo ello sugiere que se trata de un hipogonadismo que se origina en el sistema nervioso central, y que tiene una patogenia similar a la del hipogonadismo que se observa en otros procesos que cursan con pérdida de peso. El tratamiento con andrógenos en varones y con estrógenos en mujeres es parcialmente eficaz, pero lo ideal para los pacientes afectados es el adecuado control de la infección por el VIH (74). troviral Therapy for HIV-1 Infection: Recommendations of an International AIDS Society-USA Panel. J Acquir Immune Defic Syndr 2002; 31: 257-75.

5. Grunfeld C, Pang M, Doerrler W, Shigenaga JK, Jensen P, Feingold KR. Lipids, lipoproteins, triglyceride clearance and cytokines in human immunodeficiency virus infection and the acquired immunodeficiency syndrome. J Clin Endocrinol Metab 1992; 74: 1045-52.

6. Carr A, Samaras K, Burton S, Law M, Freund J, Chisholm DJ, Cooper DA. A syndrome of peripheral lipodystrophy, hyperlipidaemia and insulin resistance in patients receiving HIV protease inhibitors. AIDS 1998; 12: F51-F58. 
7. Cheseaux JJ, Jotterand V, Aebi C, Gnehm H, Kind C, Nadal D, et al. Hyperlipidemia in HIV-infected children treated with protease inhibitors: relevance for cardiovascular diseases. J Acquir Immune Defic Syndr 2002; 30: 288-93.

8. Chang ES, Tetreault DD, Liu YT, Beall GN. The effects of antiretroviral protease inhibitors on serum lipid levels in HIV-infected patients. J Am Diet Assoc 2001; 101: 687-9.

9. Mulligan K, Grunfeld C, Tai VW, Algren H, Pang M, Chernoff DN et al. Hyperlipidemia and insulin resistance are induced by protease inhibitors independent of changes in body composition in patients with HIV infection. J Acquir Immune Defic Syndr Hum Retrovirol 2000; 23: 3543.

10. Segarra-Newnham M. Hyperlipidemia in HIV-positive patients receiving antiretrovirals. Ann Pharmacother 2002; 36: 592-5.

11. National Cholesterol Education Program. Executive Summary of the Third Report of the National Cholesterol Education Program (NCEP) Expert Panel on Detection, Evaluation, and Treatment of High Blood Cholesterol in Adults (Adult Treatment Panel III). JAMA 2001; 285: 2486-97.

12. Dubé MP, Sprecher D, Henry WK, Aberg JA, Torriani FJ, Hodis HN, et al. Preliminary guidelines for the evaluation and management of dyslipidemia in HIV-infected adults receiving antiretroviral therapy: recommendations of the adult ACTG cardiovascular disease focus group. Clin Infect Dis 2000; 31: 1216-24.

13. Saag MS, Powderly WG, Schambelan M, Benson CA, Carr A, Currier JS, et al. Switching antiretroviral drugs for treatment of metabolic complications in HIV-1 infection: summary of selected trials. Topics HIV Med 2002; 10: 47-51.

14. Knopp RH. Drug treatment of lipid disorders. N Engl J Med 1999; 341: 498-510.

15. Geletko SM, Zuwallack AR. Treatment of Hyperlipidemia in HIVinfected Patients. Am J Health Syst Pharm 2001; 58: 607-14.

16. Barreiro P, Soriano V, Blanco F. Risks and benefits of replacing protease inhibitors by nevirapine in HIV-infected subjects under long-term successful triple combination therapy. AIDS 2000; 14: 807-12.

17. Falusi OM, Aberg JA. HIV and Cardiovascular Risk Factors. The AIDS Reader 2001; 11: 263-8.

18. Hadigan C, Meigs JB, Corcoran C, Rietschel P, Piecuch S, Basgoz N, et al. Metabolic abnormalities and cardiovascular disease risk factors in adults with human immunodeficiency virus infection and lipodystrophy. Clin Infect Dis 2001; 32: 130-9.

19. Gotto AM Jr. Management of dyslipidemia. Am J Med 2002; 112 Supl 8A: 10S-18S.

20. Zambón D, Sabaté J, Muñoz S, Campero B, Casals E, Merlos M et al. Substituting walnuts for monounsaturated fat improves the serum lipid profile of hypercholesterolemic men and women. Ann Intern Med. 2000; 132: 538-46.

21. Halbert JA, Silagy CA, Finucane P, Withers RT, Hamdorf PA. Exercise training and blood lipids in hyperlipidemic and normolipidemic adults: a meta-analysis of randomized, controlled trials. Eur J Clin Nutr 1999; 53: 514-22.

22. Long-Term Investigation with Pravastatin in Ischemic Disease (LIPID) Study Group. Prevention of cardiovascular events and death with pravastatin in patients with coronary heart disease and a broad range of initial cholesterol levels. N Engl J Med 1998; 339: 1349-57.

23. Panel on Clinical Practices for the Treatment of HIV Infection. Guidelines for the use of antiretroviral agents in HIV-infected adults and adolescents. Ann Intern Med 1998; 128: 1079-100. (Updated 2002 February 4, disponible en http://aidsinfo.nih.gov).

24. Mastroianni CM, d'Ettorre G, Forcina G. Rhabdomyolysis after cerivastatin-gemfibrozil therapy in an HIV-infected patient with protease inhibitor-related hyperlipidemia. AIDS 2001; 15: 820-1.

25. Roca B, Calvo B, Monferrer R. Severe Rhabdomyolysis and CerivastatinGemfibrozil Combination Therapy. Ann Pharmacother 2002; 36: 730-1.

26. Calza L, Manfredi R, Chiodo F. Use of fibrates in the management of hyperlipidemia in HIV-infected patients receiving HAART. Infection 2002; 30: 26-31.

27. Green ML. Evaluation and Management of Dyslipidemia in Patients with HIV Infection. J Gen Intern Med 2002; 17: 797-810.

28. The American Association of Clinical Endocrinologists medical guidelines for clinical practice for the diagnosis and treatment of dyslipidemia and prevention of atherogenesis. Endocr Pract 2000; 6: 162-213.

29. Lichtenstein KA, Ward DJ, Moorman AC, Delaney KM, Young B, Palella FJ Jr. Clinical assessment of HIV-associated lipodystrophy in an ambulatory population. AIDS 2001; 15: 1389-98.
30. Reus S, Arroyo E, Boix V, Portilla J. Lipodistrofia e hiperglucemia producidas por inhibidores de la proteasa. An Med Interna (Madrid) 2000; 17: $123-6$.

31. Carr A, Miller J, Law M, Cooper DA. A syndrome of lipoatrophy, lactic acidaemia and liver dysfunction associated with HIV nucleoside analogue therapy: contribution to protease inhibitor-related lipodystrophy syndrome. AIDS 2000; 14: F25-F32.

32. Mallal SA, John M, Moore CB, James IR, McKinnon EJ. Contribution of nucleoside analogue reverse transcriptase inhibitors to subcutaneous fat wasting in patients with HIV infection. AIDS 2000; 14: 1309-16.

33. Maher B, Afirevic A, Vilar FJ, Wilkins EG, Park BK, Pirmohamed M. TNF-alpha promoter region gene polymorphisms in HIV-positive patients with lipodystrophy. AIDS 2002; 16: 2013-8.

34. Christeff N, Melchior JC, De Truchis P, Perronne C, Gougen ML. Increased serum interferon alpha in HIV-1 associated lipodystrophy syndrome. Eur J Clin Invest 2002; 32: 43-50.

35. Purnell JQ, Zambon A, Knopp RH, Pizzuti DJ, Achari R, Leonard JM et al. Effect of ritonavir on lipids and post-heparin lipase in normal subjects. AIDS 2000; 14: 51-7.

36. Caron M, Auclair M, Vigouroux C, Glorian M, Forest C, Capeau J. The HIV protease inhibitor indinavir impairs sterol regulatory element-binding protein-1 intranuclear localization, inhibits preadipocyte differentiation, and induces insulin resistance. Diabetes 2001; 50: 1378-88.

37. Kakuda T, Brundage R, Anderson P, Fletcher C. Nucleoside reverse transcriptase inhibitor-induced mitochondrial toxicity as an etiology for lipodystrophy. AIDS 1999; 13: 2311-2.

38. Schoen RE, Thaete FL, Sankey SS, Weissfeld JL, Kuller LH. Sagittal diameter in comparison with single slice CT as a predictor of total visceral adipose tissue volume. Int J Obes Relat Metab Disord 1998; 22: 338-42.

39. Kotler DP, Thea DM, Heo M, Allison DB, Engelson ES, Wang J, et al. Relative influences of sex, race, environment, and HIV infection on body composition in adults. Am J Clin Nutr 1999; 69: 432-9.

40. Rankinen T, Kim SY, Perusse L, Despres JP, Bouchard C. The prediction of abdominal visceral fat level from body composition and anthropometry: ROC analysis. Int J Obes Relat Metab Disord 1999; 23: 801-9.

41. Gervasoni C, Ridolfo AL, Trifiro G, Santambrogio S, Norbiato G, Musicco $\mathrm{M}$ et al. Redistribution of body fat in HIV-infected women undergoing combined antiretroviral therapy. AIDS 1999; 13: 465-71.

42. Engelson ES, Glesby MJ, Mendez D, Albu JB, Wang J, Heymsfield SB, Kotler DP. Effect of recombinant human growth hormone in the treatment of visceral fat accumulation in HIV infection. J Acquir Immune Defic Syndr 2002; 30: 379-91.

43. Martínez E, García-Viejo MA, Blanch J, Gatell JM. Lipodystrophy syndrome in patients with HIV infection: Quality of life issues. Drug Saf 2001; 24: 157-66.

44. Carr A, Hudson J, Chuah J, Mallal S, Law M, Hoy J et al. HIV protease inhibitor substitution in patients with lipodystrophy: a randomized, controlled, open-label, multicentre study. AIDS 2001; 15: 1811-22.

45. Carr A, Workman C, Smith DE, Hudson J, Doong N, Martin A et al. Abacavir substitution for nucleoside analogs in patients with HIV lipoatrophy: a randomized trial. JAMA 2002; 288: 207-15.

46. Kotler DP, Rosenbaum K, Wang J, Pierson RN. Studies of body composition and fat distribution in HIV-infected and control subjects. J Acquir Immune Defic Syndr Hum Retrovirol 1999; 20: 228-37.

47. Roubenoff R, Weiss L, McDermott A, Heflin T, Cloutier GJ, Wood M, Gorbach S.. A pilot study of exercise training to reduce trunk fat in adults with HIV-associated fat redistribution. AIDS 1999; 13: 1373-5

48. Hadigan C, Corcoran C, Stanley T, Piecuch S, Klibanski A, Grinspoon $\mathrm{S}$. Fasting Hyperinsulinemia in human immunodeficiency virus-infected men: relationship to body composition, gonadal function and protease inhibitor use. J Clin Endocrinol Metab 2000; 85: 35-41.

49. Currier J, Carpenter C, Daar E, Kotler D, Wanke C. Identifying and managing morphologic complications of HIV and HAART. AIDS Read 2002; 12: 114-9.

50. Kilby JM, Tabereaux PB. Severe hyperglycemia in an HIV clinic: preexisting versus drug-associated diabetes mellitus. J Acquir Immune Defic Syndr Hum Retrovirol 1998; 17: 46-50.

51. Noor MA, Lo JC, Mulligan K, Schwarz JM, Halvorsen RA, Schambelan $\mathrm{M}$ et al. Metabolic effects of indinavir in healthy HIV-seronegative men. AIDS 2001; 15: F11-F18.

52. Miserez AR, Muller PY, Spaniol V. Indinavir inhibits sterolregulatory element-binding protein-1c- dependent lipoprotein lipase and fatty acid synthase gene activations. AIDS 2002; 16: 1587-94. 
53. Meigs JB, Mittleman MA, Nathan DM, Tofler GH, Singer DE, Murphy-Sheehy PM et al. Hyperinsulinemia, hyperglycemia, and impaired hemostasis: the Framingham Offspring Study. JAMA 2000; 283: 221-8.

54. Yeni PG, Hammer SM, Carpenter CC, Cooper DA, Fischl MA, Gatell JM et al. Antiretroviral treatment of adult HIV-1 infection in 2002: updated recommendations of the International AIDS Society-USA panel. JAMA 2002; 288: 222-35.

55. American Diabetes Association. Standards of medical care of patients with diabetes mellitus. Diabetes Care 2000; 23 (Supl. 1): S32-S42.

56. Gelato M, Mynarcik DC, McNurlan MA. Rosiglitazone mediated restoration of subcutaneous fat and improvement in insulin sensitivity in HIV-associated lipodystrophy. J Invest Med 2002; 50: 177A.

57. John M, Mallal S. Hyperlactatemia syndromes in people with HIV infection. Curr Opin Infect Dis 2002; 15: 23-9.

58. Kirpichnikov D, McFarlane SI, Sowers JR. Metformin: an update. Ann Intern Med 2002; 137: 25-33.

59. O'Moore-Sullivan TM, Prins JB. Thiazolidinediones and type 2 diabetes: new drugs for an old disease. Med J Aust 2002; 176: 381-6.

60. Gerard Y, Maulin L, Yazdanpanah Y, De La Tribonniere X, Amiel C, Maurage CA et al. Symptomatic hyperlactataemia: an emerging complication of antiretroviral therapy. AIDS 2000; 14: 2723-30.

61. Boubaker K, Flepp M, Sudre P, Furrer H, Haensel A, Hirschel B et al. Hyperlactatemia and antiretroviral therapy: the Swiss HIV Cohort Study. Clin Infect Dis 2001; 33: 1931-7.

62. Lafeuillade A, Hittinger G, Chadapaud S. Increased mitochondrial toxicity with ribavirin in HIV/HCV coinfection. Lancet 2001; 357: 280-1.

63. Moyle G. Clinical manifestations and management of antiretroviral nucleoside analog-related mitochondrial toxicity. Clin Ther 2000; 22: 911-36.

64. John M, Moore CB, James IR, Nolan D, Upton RP, McKinnon EJ,
Mallal SA. Chronic hyperlactatemia in HIV-infected patients taking antiretroviral therapy. AIDS 2001; 15: 717-23.

65. Tantisiriwat W, Tebas P, Polish LB, Casabar E, Powderly WG, Fichtenbaum CJ. Elevated lactate levels in hospitalized persons with HIV infection. AIDS Res Hum Retroviruses 2001; 17: 195-201.

66. Miller KD, Masur H, Jones EC, Joe GO, Rick ME, Kelly GG et al. High prevalence of osteonecrosis of the femoral head in HIV-infected adults. Ann Intern Med 2002; 137: 17-25.

67. Blacksin MF, Kloser PC, Simon J. Avascular necrosis of bone in human immunodeficiency virus infected patients. Clin Imaging 1999; 23: 3148.

68. Huang JS, Rietschel P, Hadigan CM, Rosenthal DI, Grinspoon S. Increased abdominal visceral fat is associated with reduced bone density in HIV infected men with lipodystrophy. AIDS 2001; 15: 975-82.

69. Tebas P, Powderly WG, Claxton S, Marin D, Tantisiriwat W, Teitelbaum SL, Yarasheski KE.. Accelerated bone mineral loss in HIV-infected patients receiving potent antiretroviral therapy. AIDS 2000; 14: F63-F67.

70. Stephens EA, Das R, Madge S, Barter J, Johnson MA. Symptomatic osteoporosis in two young HIV-positive African women. AIDS 1999; 13: 2605-6.

71. De Luis DA, Bachiller P, Izaola O, Eiros JM, Aller R. Estado nutricional de pacientes infectados por el virus de la inmunodeficiencia humana (VIH). An Med Interna (Madrid) 2001; 18: 619-23.

72. López-Herce JA. Alteraciones nutricionales en la infección por el virus de la immunodeficiència humana (VIH). An Med Interna (Madrid) 2001; 18: 617-8.

73. Strawford A, Hellerstein MK. Metabolic Complications of HIV and AIDS. Curr Infect Dis Rep 2001; 3: 183-92.

74. Dobs A, Brown T. Metabolic Abnormalities in HIV Disease and Injection Drug Use. J Acquir Immune Defic Syndr 2002; 31 Supl 2: S70-S77. 